\title{
A Certain Family of Integral Operators Associated with the Struve Functions
}

\author{
Shahid Mahmood ${ }^{1}$, H. M. Srivastava ${ }^{2,3}{ }^{(D)}$, Sarfraz Nawaz Malik ${ }^{4, *(\mathbb{D},}$, Mohsan Raza ${ }^{5}$, \\ Neelam Shahzadi ${ }^{4}$ and Saira Zainab ${ }^{6}$ \\ 1 Department of Mechanical Engineering, Sarhad University of Science and I.T, Ring Road, Peshawar 25000, \\ Pakistan; shahidmahmood757@gmail.com \\ 2 Department of Mathematics and Statistics, University of Victoria, Victoria, BC V8W 3R4, Canada; \\ harimsri@math.uvic.ca \\ 3 Department of Medical Research, China Medical University Hospital, China Medical University, \\ Taichung 40402, Taiwan \\ 4 Department of Mathematics, COMSATS University Islamabad, Wah Campus 47040, Pakistan; \\ nshahzadi356@gmail.com \\ 5 Department of Mathematics, Government College University Faisalabad, Faisalabad 38000, Pakistan; \\ mohsan976@yahoo.com \\ 6 Department of Mathematics, University of Wah, Wah Cantt 47040, Pakistan; sairazainab07@yahoo.com \\ * Correspondence: snmalik110@yahoo.com
}

Received: 31 January 2019; Accepted: 21 March 2019; Published: 2 April 2019

\begin{abstract}
This article presents the study of Struve functions and certain integral operators associated with the Struve functions. It contains the investigation of certain geometric properties like the strong starlikeness and strong convexity of the Struve functions. It also includes the criteria of univalence for a family of certain integral operators associated with the generalized Struve functions. The starlikeness and uniform convexity of the said integral operators are also part of this research.
\end{abstract}

Keywords: analytic functions; convex functions; starlike functions; strongly convex functions; strongly starlike functions; uniformly convex functions; Struve functions

MSC: $30 \mathrm{C} 45 ; 30 \mathrm{C} 50$

\section{Introduction}

We denote by $\mathcal{A}$ the class of functions $f$ that are analytic in the open unit disc $\mathcal{D}=\{z:|z|<1\}$ and of the form:

$$
f(z)=z+\sum_{n=2}^{\infty} a_{n} z^{n}
$$

Let $\mathcal{S}$ denote the class of all functions in $\mathcal{A}$, which are univalent in $\mathcal{D}$. Let $\mathcal{S}^{*}(\alpha), \tilde{\mathcal{S}}^{*}(\alpha)$ and $\tilde{\mathcal{C}}(\alpha)$ denote the classes of starlike, strongly starlike and strongly convex functions of order $\alpha$, respectively, and defined as:

$$
\begin{aligned}
& \mathcal{S}^{*}(\alpha)=\left\{f: f \in \mathcal{A} \text { and } \Re\left(\frac{z f^{\prime}(z)}{f(z)}\right)>\alpha, z \in \mathcal{U}, \alpha \in[0,1)\right\}, \\
& \tilde{\mathcal{S}}^{*}(\alpha)=\left\{f: f \in \mathcal{A} \text { and }\left|\arg \left(\frac{z f^{\prime}(z)}{f(z)}\right)\right|<\frac{\alpha \pi}{2}, z \in \mathcal{U}, \alpha \in[0,1)\right\},
\end{aligned}
$$

and:

$$
\tilde{\mathcal{C}}(\alpha)=\left\{f: f \in \mathcal{A} \text { and }\left|\arg \left(1+\frac{z f^{\prime \prime}(z)}{f^{\prime}(z)}\right)\right|<\frac{\alpha \pi}{2}, z \in \mathcal{U}, \alpha \in[0,1)\right\} .
$$


It is clear that:

$$
\tilde{\mathcal{S}}^{*}(1)=\mathcal{S}^{*}(0)=\mathcal{S}^{*}, \widetilde{\mathcal{C}}(1)=\mathcal{C}(0)=\mathcal{C}
$$

The class $\mathcal{U C V}$ of uniformly convex functions is defined as:

$$
\mathcal{U C V}=\left\{f \in \mathcal{A}: \Re\left(1+\frac{z f^{\prime \prime}(z)}{f^{\prime}(z)}\right)>\left|\frac{z f^{\prime \prime}(z)}{f^{\prime}(z)}\right|, z \in \mathcal{D}\right\} .
$$

For more detail, see [1]. If $f$ and $g$ are analytic functions, then the function $f$ is said to be subordinate to $g$, written as $f(z) \prec g(z)$, if there exists a Schwarz function $w$ with $w(0)=0$ and $|w|<1$ such that $f(z)=g(w(z))$. Furthermore, if the function $g$ is univalent in $\mathcal{U}$, then we have the following equivalent relation:

$$
f(z) \prec g(z) \Longleftrightarrow f(0)=g(0) \text { and } f(\mathcal{U}) \subset g(\mathcal{U}) .
$$

Now, we consider the second order inhomogeneous differential equation:

$$
z^{2} w^{\prime \prime}(z)+z w^{\prime}(z)+\left(z^{2}-L^{2}\right) w(z)=\frac{4\left(\frac{z}{2}\right)^{L+1}}{\sqrt{\pi} \Gamma\left(L+\frac{1}{2}\right)} .
$$

The solution of the homogeneous part is Bessel functions of order $L$, where $L$ is a real or complex number. For more details about Bessel functions, we refer to [2-8]. The particular solution of the inhomogeneous equation defined in Equation (2) is called the Struve function of order $L$; see [9]. It is defined as:

$$
X_{L}(z)=\sum_{n=0}^{\infty} \frac{(-1)^{n}(z / 2)^{2 n+L+1}}{\Gamma(n+3 / 2) \Gamma(L+n+3 / 2)} .
$$

Now, we consider the differential equation:

$$
z^{2} w^{\prime \prime}(z)+z w^{\prime}(z)-\left(z^{2}+L^{2}\right) w(z)=\frac{4\left(\frac{z}{2}\right)^{L+1}}{\sqrt{\pi} \Gamma\left(L+\frac{1}{2}\right)} .
$$

The Equation (4) differs from the Equation (2) in the coefficients of $w$. Its particular solution is called the modified Struve functions of order $L$ and is given as:

$$
Y_{L}(z)=-i e^{-i p \pi / 2} X_{L}(i z)=\sum_{n=0}^{\infty} \frac{\left(\frac{z}{2}\right)^{2 n+L+1}}{\Gamma(n+3 / 2) \Gamma\left(L+n+\frac{3}{2}\right)} .
$$

Again, consider the second order inhomogeneous differential equation:

$$
z^{2} w^{\prime \prime}(z)+b z w^{\prime}(z)+\left[c z^{2}-L^{2}+(1-b) L\right] w(z)=\frac{4\left(\frac{z}{2}\right)^{L+1}}{\sqrt{\pi} \Gamma\left(L+\frac{b}{2}\right)},
$$

where $b, c, L \in \mathbb{C}$. The Equation (5) generalizes the Equations (2) and (4). In particular, for $b=1$, $c=1$, we obtain Equation (2), and for $b=1, c=-1$, we obtain Equation (4). Its particular solution has the series form:

$$
w_{L, b, c}(z)=\sum_{n=0}^{\infty} \frac{(-1)^{n} c^{n}(z / 2)^{2 n+L+1}}{\Gamma(n+3 / 2) \Gamma(L+n+(b+2) / 2)}
$$


and is called the generalized Struve function of order $L$. This series is convergent everywhere. We take the transformation:

$$
u_{L, b, c}(z)=2^{L} \sqrt{\pi} \Gamma(L+(b+2) / 2) z^{(-L-1) / 2} w_{L, b, c}(\sqrt{z})=\sum_{n=0}^{\infty} \frac{(-c / 4)^{n} z^{n}}{(3 / 2)_{n}(q)_{n}},
$$

where $q=L+(b+2) / 2 \neq 0,-1,-2, \ldots$ and $(\gamma)_{n}=\frac{\Gamma(\gamma+n)}{\Gamma(\gamma)}=\gamma(\gamma+1) \ldots(\gamma+n-1)$. This function is analytic in the whole complex plane and satisfies the differential equation:

$$
4 z^{2} w^{\prime \prime}(z)+2(2 p+b+3) z w^{\prime}(z)+[c z+2 p+b] w(z)=2 p+b,
$$

where $\Gamma$ (.) denotes the Gamma function. The function $u_{L, b, c}$ unifies the Struve functions and modified Struve functions. The function $u_{L, b, c}$ is not in the class $\mathcal{A}$ of analytic functions; therefore, we consider the following normalized form of the Struve function as:

$$
v_{L, b, c}(z)=z u_{L, b, c}=z+\sum_{n=1}^{\infty} \frac{(-c / 4)^{n} z^{n+1}}{(3 / 2)_{n}(q)_{n}} .
$$

\section{Special cases:}

(i) For $b=1, c=1$, we have the normalized Struve function $\mathcal{X}_{L}: \mathcal{A} \rightarrow \mathcal{A}$ of order $L$. It is given as:

$$
\begin{aligned}
\mathcal{X}_{L}(z) & =2^{L} \sqrt{\pi} \Gamma\left(L+\frac{3}{2}\right) z^{\frac{(-L+1)}{2}} X_{L}(\sqrt{z}) \\
& =z+\sum_{n=1}^{\infty} \frac{(-1 / 4)^{n} z^{n+1}}{(3 / 2)_{n}(q)_{n}} .
\end{aligned}
$$

(ii) For $b=1, c=-1$, we have the normalized Struve function $\mathcal{Y}_{L}: \mathcal{A} \rightarrow \mathcal{A}$ of order $L$. It is given as:

$$
\begin{aligned}
\mathcal{Y}_{L}(z) & =2^{L} \sqrt{\pi} \Gamma\left(L+\frac{3}{2}\right) z^{\frac{(-L+1)}{2}} Y_{L}(\sqrt{z}) \\
& =z+\sum_{n=1}^{\infty} \frac{(1 / 4)^{n} z^{n+1}}{(3 / 2)_{n}(q)_{n}} .
\end{aligned}
$$

The functions $u_{L, b, c}$ and $v_{L, b, c}$ were introduced and studied by Orhan and Yugmur [10] and further investigated by other authors [11-13]. In the last few years, many mathematicians have set the univalence criteria of several of those integral operators that preserve the class $\mathcal{S}$. By using a variety of different analytic techniques, operators and special functions, several authors have studied the univalence criterion. Recently Din et al. [14] studied the univalence of integral operators involving generalized Struve functions. These operators are defined as follows:

$$
\begin{aligned}
& \mathcal{F}_{\alpha_{1}, \ldots, \alpha_{n}, \beta}(z)=\left[\beta \int_{0}^{z} t^{\beta-1} \prod_{i=1}^{n}\left(\frac{v_{L_{i}, b, c}(t)}{t}\right)^{\frac{1}{\alpha_{i}}} d t\right]^{\frac{1}{\beta}}, \\
& \mathcal{M}_{n, \gamma}(z)=\left[(n \gamma+1) \int_{0}^{z} \prod_{i=1}^{n}\left\{v_{L_{i}, b, c}(t)\right\}^{\gamma} d t\right]^{\frac{1}{n \gamma+1}},
\end{aligned}
$$


and:

$$
\mathcal{Z}_{\lambda}(z)=\left[\lambda \int_{0}^{z} t^{\lambda-1}\left(e^{v_{L_{i}, b, c}(t)}\right)^{\lambda} d t\right]^{1 / \lambda} .
$$

Now, we introduce the following integral operators $H_{L i, b, c, \gamma_{1, \ldots,}, \gamma_{n}, \beta}, I_{L i b, b, \gamma_{1}, \ldots, \gamma_{n}, \delta, \beta}: \mathcal{A} \rightarrow \mathcal{A}$ involving the generalized Struve functions as:

$$
\begin{aligned}
H_{L_{i}, b, c, \gamma_{i}, \beta}(z) & =\left\{\beta \int_{0}^{z} t^{\beta-1} \prod_{i=1}^{n}\left(\frac{v_{L_{i}, b, c}(t)}{g_{i}(t)}\right)^{\gamma_{i}} d t\right\}^{\frac{1}{\beta}}, \\
I_{L i, b, c, \gamma_{i}, \delta_{i}, \beta}(z) & =\left\{\beta \int_{0}^{z} t^{\beta-1} \prod_{i=1}^{n}\left(\frac{v_{L_{i}, b, c}^{\prime}(t)}{t}\right)^{\gamma_{i}}\left(g_{i}^{\prime}(t)\right)^{\delta_{i}} d t\right\}^{\frac{1}{\beta}},
\end{aligned}
$$

where $\gamma_{i}, \delta_{i}, \beta$ are nonzero complex numbers, $L_{i} \in \mathbb{R}$ for all $i=1,2, \cdots, n$ and $g_{i} \in \mathcal{A}$.

In this paper, our aim is to study certain geometric properties like the strong starlikeness and strong convexity of the Struve functions and univalence for the integral operators $H_{L_{i}, b, c, \gamma_{i}, \beta}$ and $I_{L_{i}, b, c, \gamma_{i}, \delta_{i}, \beta}$ associated with the generalized Struve functions. The starlikeness and uniform convexity of the said integral operators are also part of this research.

\section{Preliminary Results}

We need the following lemmas to prove our main results.

Lemma 1 ([15]). Let $G(z)$ be convex and univalent in the open unit disc with condition $G(0)=1$. Let $F(z)$ be analytic in the open unit disc with condition $F(0)=1$ and $F \prec G$ in the open unit disc. Then, $\forall n \in \mathbb{N} \cup\{0\}$, we obtain:

$$
(n+1) z^{-1-n} \int_{0}^{z} t^{n} F(t) d t \prec(n+1) z^{-1-n} \int_{0}^{z} t^{n} G(t) d t .
$$

Lemma 2 ([16]). If $g \in \mathcal{A}$ satisfies:

$$
\left|1+\frac{z g^{\prime \prime}(z)}{g^{\prime}(z)}\right|<2, \quad \text { then } g \in \mathcal{S}^{*} .
$$

Lemma 3 ([17]). If $g \in \mathcal{A}$ satisfies:

$$
\left|\frac{z g^{\prime \prime}(z)}{g^{\prime}(z)}\right|<\frac{1}{2}, \quad \text { then } g \in \mathcal{U C V} \text {. }
$$

Lemma 4 ([10]). If $b, L \in \mathbb{R}$ and $c \in \mathbb{C}, q=L+\frac{b+2}{2}$ are so constrained that $q>\max \left\{0, \frac{7|c|}{24}\right\}$, then the function $v_{L, b, c}: \mathcal{D} \longrightarrow \mathbb{C}$ satisfies the following inequalities.

(i) $\quad\left|\frac{z v_{L, b, c}^{\prime}(z)}{v_{L, b, c}(z)}-1\right| \leq \frac{|c|(6 q-|c|)}{3(4 q-|c|)(3 q-|c|)}$,

(ii) $\quad\left|\frac{z v_{L, b, c}^{\prime \prime}(z)}{v_{L, b, c}^{\prime}(z)}\right| \leq \frac{6|c|}{(12 q-7|c|)}$.

Lemma 5 ([18]). If $g \in \mathcal{A}$ satisfies the following inequality:

$$
\frac{1-|z|^{2 \Re(\alpha)}}{\Re(\alpha)}\left|\frac{z g^{\prime \prime}(z)}{g^{\prime}(z)}\right| \leq 1, \Re(\alpha)>0,
$$


then for every complex number $\beta, \Re \beta \geq \Re(\alpha)$, the function:

$$
G_{\beta}(z)=\left(\beta \int_{0}^{z} t^{\beta-1} g^{\prime}(t) d t\right)^{\frac{1}{\beta}} \in \mathcal{S} .
$$

Lemma 6 ([19]). Let $g(z)=z+a_{2} z^{2}+\cdots$ be the analytic function in $\mathcal{D}$. If:

$$
\left|\frac{g^{\prime \prime}(z)}{g^{\prime}(z)}\right| \leq K, \quad z \in \mathcal{D}
$$

where $K \simeq 3.05$, then $g$ is univalent in $\mathcal{D}$.

Remark 1. The constant $K$ is the solution of the equation $8\left[x(x-2)^{3}\right]^{\frac{1}{2}}-3(4-x)^{2}=12$. An approximation by using the computer programs suggest the value 3.03902118847875 . Kudriasov used the approximated value equal to 3.05 .

\section{Geometric Properties of Generalized Struve Functions}

Theorem 1. If $q \geq \frac{7|c|}{12}$, then $v_{L, b, c} \in \tilde{\mathcal{S}}^{*}(\alpha)$, where:

$$
\alpha=\frac{2}{\pi} \arcsin \left(\psi \sqrt{1-\frac{\psi^{2}}{4}}+\frac{\psi}{2} \sqrt{1-\psi^{2}}\right)
$$

and $\psi=\frac{4|c|}{3(4 q-|c|)}$ is such that $\arcsin \frac{\psi}{2}+\arcsin \psi \in\left[-\frac{\pi}{2}, \frac{\pi}{2}\right]$.

Proof. By using Equation (8) with the triangle inequality, we have:

$$
\left|v_{L, b, c}^{\prime}(z)-1\right| \leq \sum_{n=1}^{\infty} \frac{|c|^{n}(n+1)}{(3 / 2)_{n} 4^{n}(q)_{n}}
$$

By the help of the inequalities:

$$
(3 / 2)_{n} \geq \frac{3}{4}(n+1),(q)_{n} \geq q^{n}, \forall n \geq 1,
$$

we obtain:

$$
\begin{aligned}
\left|v_{L, b, c}^{\prime}(z)-1\right| & \leq \frac{|c|}{3 q} \sum_{n=1}^{\infty}\left(\frac{|c|}{4 q}\right)^{n-1} \\
& =\frac{4|c|}{3(4 q-|c|)}=\psi, \quad q>\frac{|c|}{4}
\end{aligned}
$$

For $q \geq \frac{7|c|}{12}$, it is clear that $0<\psi \leq 1$. Furthermore, from expression (17), we concluded that:

$$
v_{L, b, c}^{\prime}(z) \prec 1+\psi z \Rightarrow\left|\arg \left(v_{L, b, c}^{\prime}(z)\right)\right|<\arcsin \psi .
$$

With the help of Lemma 1, take $n=0$ with $F(z)=v_{L, b, c}^{\prime}(z)$ and $G(z)=1+\psi z$, and we get:

$$
\frac{v_{L, b, c}(z)}{z} \prec 1+\frac{\psi}{2} z .
$$


As a result:

$$
\left|\arg \left(\frac{v_{L, b, c}(z)}{z}\right)\right|<\arcsin \frac{\psi}{2} .
$$

By using relations (18) and (19), we obtain:

$$
\begin{aligned}
\left|\arg \left(\frac{z v_{L, b, c}^{\prime}(z)}{v_{L, b, c}(z)}\right)\right| & =\left|\arg \left(\frac{z}{v_{L, b, c}(z)}\right)-\arg \left(v_{L, b, c}^{\prime}(z)\right)\right| \\
& \leq\left|\arg \left(\frac{z}{v_{L, b, c}(z)}\right)\right|+\left|\arg \left(v_{L, b, c}^{\prime}(z)\right)\right| \\
& <\arcsin \frac{\psi}{2}+\arcsin \psi .
\end{aligned}
$$

As $0<\psi \leq 1$, thus one can write the above last expression as:

$$
\left|\arg \left(\frac{z v_{L, b, c}^{\prime}(z)}{v_{L, b, c}(z)}\right)\right|<\arcsin \left(\psi \sqrt{1-\frac{\psi^{2}}{4}}+\frac{\psi}{2} \sqrt{1-\psi^{2}}\right),
$$

which shows that $v_{L, b, c} \in \tilde{\mathcal{S}}^{*}(\alpha)$ for $\alpha=\frac{2}{\pi} \arcsin \left(\psi \sqrt{1-\frac{\psi^{2}}{4}}+\frac{\psi}{2} \sqrt{1-\psi^{2}}\right)$.

Theorem 2. If $q \geq \frac{4|c|}{3}$, then $v_{L, b, c} \in \tilde{\mathcal{C}}(\alpha)$, where:

$$
\alpha=\frac{2}{\pi} \arcsin \left(\varphi \sqrt{1-\frac{\varphi^{2}}{4}}+\frac{\varphi}{2} \sqrt{1-\varphi^{2}}\right),
$$

and $\varphi=\frac{2|c|}{3 q-2|c|}$ is such that $\arcsin \frac{\varphi}{2}+\arcsin \varphi \in\left[-\frac{\pi}{2}, \frac{\pi}{2}\right]$.

Proof. By using the well-known triangle inequality:

$$
\left|z_{1}+z_{2}\right| \leq\left|z_{1}\right|+\left|z_{2}\right|
$$

with the inequalities:

$$
(n+1)^{2} \leq 4^{n},(q)_{n} \geq q^{n} \quad \forall n \in \mathbb{N},
$$

we obtain:

$$
\begin{aligned}
\left|\left(z v_{L, b, c}^{\prime}(z)\right)^{\prime}-1\right| & \leq \sum_{n=1}^{\infty} \frac{|c|^{n}(n+1)^{2}}{(3 / 2)_{n} 4^{n}(q)_{n}} \\
& \leq \frac{2|c|}{3 q} \sum_{n=1}^{\infty}\left(\frac{2|c|}{3 q}\right)^{n-1} \\
& =\frac{2|c|}{3 q-2|c|}=\varphi .
\end{aligned}
$$

It is clear that $0<\varphi \leq 1$ for $q \geq \frac{4|c|}{3}$, and from the expression (22), we conclude that:

$$
\left(z v_{L, b, c}^{\prime}(z)\right)^{\prime} \prec 1+\varphi z \Rightarrow\left|\arg \left(z v_{L, b, c}^{\prime}(z)\right)^{\prime}\right|<\arcsin \varphi \text {. }
$$

With the help of Lemma 1, take $n=0$ with $F(z)=\left(z v_{L, b, c}^{\prime}(z)\right)^{\prime}$ and $G(z)=1+\varphi z$, and we get:

$$
\frac{z v_{L, b, c}^{\prime}(z)}{z} \prec 1+\frac{\varphi}{2} z \text {. }
$$


This implies that:

$$
v_{L, b, c}^{\prime}(z) \prec 1+\frac{\varphi}{2} z .
$$

As a result:

$$
\left|\arg v_{L, b, c}^{\prime}(z)\right|<\arcsin \frac{\varphi}{2} .
$$

By using relations (23) and (25), we obtain:

$$
\begin{aligned}
\left|\arg \left(\frac{\left(z v_{L, b, c}^{\prime}(z)\right)^{\prime}}{v_{L, b, c}^{\prime}(z)}\right)\right| & =\left|\arg \left(z v_{L, b, c}^{\prime}(z)\right)^{\prime}-\arg v_{L, b, c}^{\prime}(z)\right| \\
& \leq\left|\arg \left(z v_{L, b, c}^{\prime}(z)\right)^{\prime}\right|+\left|\arg \left(v_{L, b, c}^{\prime}(z)\right)\right| \\
& <\arcsin \frac{\varphi}{2}+\arcsin \varphi .
\end{aligned}
$$

As $0<\varphi \leq 1$, thus one can write the above last expression as:

$$
\left|\arg \left(\frac{\left(z v_{L, b, c}^{\prime}(z)\right)^{\prime}}{v_{L, b, c}^{\prime}(z)}\right)\right|<\arcsin \left(\varphi \sqrt{1-\frac{\varphi^{2}}{4}}+\frac{\varphi}{2} \sqrt{1-\varphi^{2}}\right)
$$

which shows that $v_{L, b, c} \in \tilde{\mathcal{C}}(\alpha)$ for $\alpha=\frac{2}{\pi} \arcsin \left(\varphi \sqrt{1-\frac{\varphi^{2}}{4}}+\frac{\varphi}{2} \sqrt{1-\varphi^{2}}\right)$.

Theorem 3. Let $q>\frac{19|c|}{12}$, then $v_{L, b, c} \in \mathcal{U C V}$.

Proof. Since:

$$
\left|\frac{z v_{L, b, c}^{\prime \prime}(z)}{v_{L, b, c}^{\prime}(z)}\right| \leq \frac{6|c|}{(12 q-7|c|)}
$$

By using Lemma 3, we have the required result.

\section{Univalence Criteria for Integral Operators}

In this section, we find the univalence of these integral operators defined by generalized Struve functions, by using the above lemmas.

Theorem 4. Let $L_{1}, \ldots, L_{n}, b \in \mathbb{R}, c \in \mathbb{C}$ and $q_{i}>\frac{7|c|}{24}$ with $q_{i}=L_{i}+\frac{b+2}{2}, i=1, \ldots, n$. Let $v_{L_{i}, b, c}: \mathcal{D} \longrightarrow \mathbb{C}$ be defined in the Equation (8). Suppose $q=\min \left(q_{1}, q_{2}, \ldots, q_{n}\right), \gamma_{i}$ are non-zero complex numbers and if $g_{i} \in \mathcal{A}$ with:

$$
\left|\frac{g_{i}^{\prime \prime}(z)}{g_{i}^{\prime}(z)}\right| \leq K, \quad z \in \mathcal{D}
$$

where $K \simeq 3.05$, these numbers satisfying the relations:

$$
\frac{1}{\Re(\alpha)}\left(1+\frac{|c|(6 q-|c|)}{3(4 q-|c|)(3 q-|c|)}\right) \sum_{i=1}^{n}\left|\gamma_{i}\right|+\frac{4}{\Re(\alpha)} \sum_{i=1}^{n}\left|\gamma_{i}\right|<1,
$$

when $0<\Re(\alpha)<1$ and for $\Re(\alpha) \geq 1$ :

$$
\frac{1}{\Re(\alpha)}\left(1+\frac{|c|(6 q-|c|)}{3(4 q-|c|)(3 q-|c|)}\right) \sum_{i=1}^{n}\left|\gamma_{i}\right|+4 \sum_{i=1}^{n}\left|\gamma_{i}\right|<1
$$

then for every complex number $\beta, \Re(\beta) \geq \Re(\alpha)>0$, the function $H_{L_{i}, b, c, \gamma_{i}, \beta}$ defined in (14) is univalent. 
Proof. Consider the function:

$$
H_{L_{i}, b, c, \gamma_{i}}(z)=\int_{0}^{z} \prod_{i=1}^{n}\left(\frac{v_{L_{i}, b, c}(t)}{g_{i}(t)}\right)^{\gamma_{i}} d t
$$

By taking the derivative of Equation (28), we get:

$$
H_{L_{i}, b, c, \gamma_{i}}^{\prime}(z)=\prod_{i=1}^{n}\left(\frac{v_{L_{i}, b, c}(z)}{g_{i}(z)}\right)^{\gamma_{i}} .
$$

It is clear that $H_{L_{i}, b, c, \gamma_{i}}(0)=H_{L_{i}, b, c, \gamma_{i}}^{\prime}(0)-1=0$. It follows easily that:

$$
\frac{z H_{L_{i}, b, c, \gamma_{i}}^{\prime \prime}(z)}{H_{L_{i}, b, c, \gamma_{i}}^{\prime}(z)}=\sum_{i=1}^{n} \gamma_{i}\left\{\left(\frac{z v_{L_{i}, b, c}^{\prime}(z)}{v_{L_{i}, b, c}(z)}\right)-\left(\frac{z g_{i}^{\prime}(z)}{g_{i}(z)}\right)\right\}
$$

and:

$$
\frac{1-|z|^{2 \Re(\alpha)}}{\Re(\alpha)}\left|\frac{z H_{L_{i}, b, c, \gamma_{i}}^{\prime \prime}(z)}{H_{L_{i}, b, c, \gamma_{i}}^{\prime}(z)}\right| \leq \frac{1-|z|^{2 \Re(\alpha)}}{\Re(\alpha)}\left\{\sum_{i=1}^{n}\left|\gamma_{i}\right|\left|\frac{z v_{L_{i}, b, c}^{\prime}(z)}{v_{L_{i}, b, c}(z)}\right|+\sum_{i=1}^{n}\left|\gamma_{i}\right|\left|\frac{z g_{i}^{\prime}(z)}{g_{i}(z)}\right|\right\} .
$$

Now, using the Lemma 6, we have $g_{i} \in \mathcal{S}, i=1, \ldots, n$, and:

$$
\left|\frac{z g_{i}^{\prime}(z)}{g_{i}(z)}\right| \leq \frac{1+|z|}{1-|z|}
$$

By virtue of the above inequality (30), we get:

$$
\begin{aligned}
\frac{1-|z|^{2 \Re(\alpha)}}{\Re(\alpha)}\left|\frac{z H_{L_{i}, b, c, \gamma_{i}}^{\prime \prime}(z)}{H_{L_{i}, b, c, \gamma_{i}}^{\prime}(z)}\right| & \leq \frac{1-|z|^{2 \Re(\alpha)}}{\Re(\alpha)}\left\{\sum_{i=1}^{n}\left|\gamma_{i}\right|\left|\frac{z v_{L_{i}, b, c}^{\prime}(z)}{v_{L_{i}, b, c}(z)}\right|+\sum_{i=1}^{n}\left|\gamma_{i}\right| \frac{1+|z|}{1-|z|}\right\} \\
& \leq \frac{1-|z|^{2 \Re(\alpha)}}{\Re(\alpha)} \sum_{i=1}^{n}\left|\gamma_{i}\right|\left|\frac{z v_{L_{i}, b, c}^{\prime}(z)}{v_{L_{i}, b, c}(z)}\right|+\frac{1-|z|^{2 \Re(\alpha)}}{\Re(\alpha)} \frac{2}{1-|z|} \sum_{i=1}^{n}\left|\gamma_{i}\right| .
\end{aligned}
$$

First, we consider the part:

$$
\frac{1-|z|^{2 \Re(\alpha)}}{\Re(\alpha)} \sum_{i=1}^{n}\left|\gamma_{i}\right|\left|\frac{z v_{L_{i}, b, c}^{\prime}(z)}{v_{L_{i}, b, c}(z)}\right|
$$

This implies that:

$$
\frac{1-|z|^{2 \Re(\alpha)}}{\Re(\alpha)} \sum_{i=1}^{n}\left|\gamma_{i}\right|\left|\frac{z v_{L_{i}, b, c}^{\prime}(z)}{v_{L_{i}, b, c}(z)}\right| \leq \frac{1}{\Re(\alpha)} \sum_{i=1}^{n}\left|\gamma_{i}\right|\left|\frac{z v_{L_{i}, b, c}^{\prime}(z)}{v_{L_{i}, b, c}(z)}\right| .
$$

Using Lemma 5, we have:

$$
\frac{1-|z|^{2 \Re(\alpha)}}{\Re(\alpha)} \sum_{i=1}^{n}\left|\gamma_{i}\right|\left|\frac{z v_{L_{i}, b, c}^{\prime}(z)}{v_{L_{i}, b, c}(z)}\right| \leq \frac{1}{\Re(\alpha)} \sum_{i=1}^{n}\left|\gamma_{i}\right|\left\{1+\frac{|c|\left(6 q_{i}-|c|\right)}{3\left(4 q_{i}-|c|\right)\left(3 q_{i}-|c|\right)}\right\} .
$$

We define the function $\tau:\left(\frac{7|c|}{24}, \infty\right) \longrightarrow \mathbb{R}, \tau(x)=\frac{|c|(6 x-|c|)}{3(4 x-|c|)(3 x-|c|)}$. It is a decreasing function; therefore:

$$
\frac{|c|\left(6 q_{i}-|c|\right)}{3\left(4 q_{i}-|c|\right)\left(3 q_{i}-|c|\right)} \leq \frac{|c|(6 q-|c|)}{3(4 q-|c|)(3 q-|c|)},
$$


hence:

$$
\frac{1-|z|^{2 \Re(\alpha)}}{\Re(\alpha)} \sum_{i=1}^{n}\left|\gamma_{i}\right| \frac{z v_{L_{i}, b, c}^{\prime}(z)}{v_{L_{i}, b, c}(z)}\left|\leq \frac{1}{\Re(\alpha)} \sum_{i=1}^{n}\right| \gamma_{i} \mid\left\{1+\frac{|c|(6 q-|c|)}{3(4 q-|c|)(3 q-|c|)}\right\}
$$

Now, we consider the part:

$$
\frac{1-|z|^{2 \Re(\alpha)}}{\Re(\alpha)} \frac{2}{1-|z|} \sum_{i=1}^{n}\left|\gamma_{i}\right|
$$

For this, we have the following cases:

(1) For $0<\Re(\alpha)<1$, then the function $v:(0,1) \longrightarrow \mathbb{R}, v(x)=1-a^{2 x}, x=\Re(\alpha)$ and $|z|=a$ is increasing and:

$$
1-|z|^{2 \Re(\alpha)} \leq 1-|z|^{2}
$$

therefore:

$$
\frac{1-|z|^{2 \Re(\alpha)}}{\Re(\alpha)} \frac{2}{1-|z|} \sum_{i=1}^{n}\left|\gamma_{i}\right| \leq \frac{4}{\Re(\alpha)} \sum_{i=1}^{n}\left|\gamma_{i}\right|
$$

From the inequalities (31) and (32), for $0<\Re(\alpha)<1$, we have:

$$
\frac{1-|z|^{2 \Re(\alpha)}}{\Re(\alpha)}\left|\frac{z H_{L_{i}, b, c, \gamma_{i}}^{\prime \prime}(z)}{H_{L_{i}, b, c, \gamma_{i}}^{\prime}(z)}\right| \leq \frac{1}{\Re(\alpha)}\left(1+\frac{|c|(6 q-|c|)}{3(4 q-|c|)(3 q-|c|)}\right) \sum_{i=1}^{n}\left|\gamma_{i}\right|+\frac{4}{\Re(\alpha)} \sum_{i=1}^{n}\left|\gamma_{i}\right| .
$$

(2) For $\Re(\alpha) \geq 1$, consider the function $w:[1, \infty) \longrightarrow \mathbb{R}, w(x)=\frac{1-a^{2 x}}{x}, x=\Re(\alpha)$ and $|z|=a$ is a decreasing function and:

$$
\frac{1-|z|^{2 \Re(\alpha)}}{\Re(\alpha)} \leq 1-|z|^{2}
$$

therefore:

$$
\frac{1-|z|^{2 \Re(\alpha)}}{\Re(\alpha)} \frac{2}{1-|z|} \sum_{i=1}^{n}\left|\gamma_{i}\right| \leq 4 \sum_{i=1}^{n}\left|\gamma_{i}\right|
$$

By combining the inequalities (31) and (34) for $\Re(\alpha) \geq 1$, we get:

$$
\left(\frac{1-|z|^{2 \Re(\alpha)}}{\Re(\alpha)}\right)\left|\frac{z H_{L_{i}, b, c, \gamma_{i}}^{\prime \prime}(z)}{H_{L_{i}, b, c, \gamma_{i}}^{\prime}(z)}\right| \leq \frac{1}{\Re(\alpha)}\left(1+\frac{|c|(6 q-|c|)}{3(4 q-|c|)(3 q-|c|)}\right) \sum_{i=1}^{n}\left|\gamma_{i}\right|+4 \sum_{i=1}^{n}\left|\gamma_{i}\right|
$$

From the inequalities (26), (27), (33) and (35), we obtain:

$$
\frac{1-|z|^{2 \Re(\alpha)}}{\Re(\alpha)}\left|\frac{z H_{L_{i}, b, c, \gamma_{i}}^{\prime \prime}(z)}{H_{L_{i}, b, c, \gamma_{i}}^{\prime}(z)}\right|<1
$$

Therefore, using Lemma 5, we get the required result.

Theorem 5. Let $L_{1}, \ldots L_{n}, b \in \mathbb{R}, c \in \mathbb{C}$ and $q_{i}>\frac{7|c|}{24}$ with $q_{i}=L_{i}+\frac{(b+2)}{2}, i=1, \ldots, n$. Let $v_{L_{i}, b, c}: \mathcal{D} \longrightarrow$ $\mathbb{C}$ be defined in the Equation (8). Suppose $q=\min \left(q_{1}, q_{2}, \ldots . . q_{n}\right), \gamma_{i}, \delta_{i}$ are non-zero complex numbers and if $g_{i} \in \mathcal{A}$ with

$$
\left|\frac{g_{i}^{\prime \prime}(z)}{g_{i}^{\prime}(z)}\right| \leq K, \quad z \in \mathcal{D}
$$

where $K \simeq 3.05$, and these numbers satisfy the relation:

$$
\frac{1}{\Re(\alpha)} \frac{|c|(6 q-|c|)}{3(4 q-|c|)(3 q-|c|)} \sum_{i=1}^{n}\left|\gamma_{i}\right|+\frac{2 K}{(2 \Re(\alpha)+1)^{\frac{(2 \Re(\alpha)+1)}{2 \Re(\alpha)}}} \sum_{i=1}^{n}\left|\delta_{i}\right|<1 .
$$


Then, for every complex number $\beta, \Re(\beta) \geq \Re(\alpha)>0$, the function $I_{L_{i}, b, c, \gamma_{i}, \delta_{i}, \beta}$ defined in Equation (15) is univalent.

Proof. Consider the function:

$$
I_{L_{i}, b, c, \gamma_{i}, \delta_{i}}(z)=\int_{0}^{z} \prod_{i=1}^{n}\left(\frac{v_{L_{i}, b, c}(t)}{t}\right)^{\gamma_{i}}\left(g_{i}^{\prime}(t)\right)^{\delta_{i}} d t .
$$

By taking the derivative of Equation (37), we get:

$$
I_{L_{i}, b, c, \gamma_{i}, \delta_{i}}^{\prime}(z)=\prod_{i=1}^{n}\left(\frac{v_{L_{i}, b, c}(z)}{z}\right)^{\gamma_{i}}\left(g_{i}^{\prime}(z)\right)^{\delta_{i}} .
$$

It is clear that $I_{L_{i}, b, c, \gamma_{i}, \delta_{i}} \in \mathcal{A}$. It follows easily that:

$$
\frac{z I_{L_{i}, b, c, \gamma_{i}, \delta_{i}}^{\prime \prime}(z)}{I_{L_{i}, b, c, \gamma_{i}, \delta_{i}}^{\prime}(z)}=\sum_{i=1}^{n} \gamma_{i}\left(\frac{z v_{L_{i}, b, c}^{\prime}(z)}{v_{L_{i}, b, c}(z)}-1\right)+\sum_{i=1}^{n} \delta_{i}\left\{\frac{z g_{i}^{\prime \prime}(z)}{g_{i}^{\prime}(z)}\right\} .
$$

Therefore, we obtain:

$$
\begin{aligned}
& \frac{1-|z|^{2 \Re(\alpha)}}{\Re(\alpha)}\left|\frac{z I_{L_{i}, b, c, \gamma_{i}, \delta_{i}}^{\prime \prime}(z)}{I_{L_{i}, b, c, \gamma_{i}, \delta_{i}}^{\prime}(z)}\right| \\
\leq & \frac{1-|z|^{2 \Re(\alpha)}}{\Re(\alpha)}\left\{\left|\gamma_{i}\right|\left|\frac{z v_{L_{i}, b, c}^{\prime}(z)}{v_{L_{i}, b, c}(z)}-1\right|+|z|\left|\delta_{i}\right|\left|\frac{g_{i}^{\prime \prime}(z)}{g_{i}^{\prime}(z)}\right|\right\} .
\end{aligned}
$$

This implies that:

$$
\begin{aligned}
\frac{1-|z|^{2 \Re(\alpha)}}{\Re(\alpha)}\left|\frac{z I_{L_{i}, b, c, \gamma_{i}, \delta i}^{\prime \prime}(z)}{I_{L_{i}, b, c, \gamma i, \delta_{i}}(z)}\right| \leq & \left\{\frac{1-|z|^{2 \Re(\alpha)}}{\Re(\alpha)} \sum_{i=1}^{n}\left|\gamma_{i}\right|\left|\frac{z v_{L_{i}, b, c}^{\prime}(z)}{v_{L_{i}, b, c}(z)}-1\right|\right. \\
& \left.+\frac{1-|z|^{2 \Re(\alpha)}}{\Re(\alpha)}|z| \sum_{i=1}^{n}\left|\delta_{i}\right|\left|\frac{g_{i}^{\prime \prime}(z)}{g_{i}^{\prime}(z)}\right|\right\} .
\end{aligned}
$$

Using Lemmas 4 and 6, we get:

$$
\begin{aligned}
\frac{1-|z|^{2 \Re(\alpha)}}{\Re(\alpha)}\left|\frac{z I_{L_{i}, b, c, \gamma_{i}, \delta_{i}}^{\prime \prime}(z)}{I_{L_{i}, b, c, \gamma_{i}, \delta_{i}}^{\prime}(z)}\right| \leq & \left\{\frac{1-|z|^{2 \Re(\alpha)}}{\Re(\alpha)} \sum_{i=1}^{n}\left|\gamma_{i}\right| \frac{|c|\left(6 q_{i}-|c|\right)}{3\left(4 q_{i}-|c|\right)\left(3 q_{i}-|c|\right)}\right. \\
& \left.+\frac{1-|z|^{2 \Re(\alpha)}}{\Re(\alpha)}|z| K \sum_{i=1}^{n}\right\}\left|\delta_{i}\right| .
\end{aligned}
$$

As was mentioned before:

$$
\frac{|c|\left(6 q_{i}-|c|\right)}{3\left(4 q_{i}-|c|\right)\left(3 q_{i}-|c|\right)} \leq \frac{|c|(6 q-|c|)}{3(4 q-|c|)(3 q-|c|)} ;
$$

therefore:

$$
\begin{aligned}
\frac{1-|z|^{2 \Re(\alpha)}}{\Re(\alpha)}\left|\frac{z I_{L_{i}, b, c, \gamma_{i}, \delta_{i}}^{\prime \prime}(z)}{I_{L_{i}, b, c, \gamma_{i}, \delta_{i}}(z)}\right| \leq & \left\{\frac{1-|z|^{2 \Re(\alpha)}}{\Re(\alpha)} \frac{|c|(6 q-|c|)}{3(4 q-|c|)(3 q-|c|)} \sum_{i=1}^{n}\left|\gamma_{i}\right|\right. \\
& \left.+\frac{1-|z|^{2 \Re(\alpha)}}{\Re(\alpha)}|z| K \sum_{i=1}^{n}\left|\delta_{i}\right|\right\} .
\end{aligned}
$$


Consider the function $h:[0,1] \longrightarrow \mathbb{R}, h(x)=\frac{x\left(1-x^{2 a}\right)}{a}, x=|z|, a=\Re(\alpha)$. Then:

$$
\operatorname{maxh}(x)=\frac{2}{(2 a+1)^{\frac{(2 a+1)}{2 a}}}, x \in[0,1] .
$$

This implies that:

$$
\begin{aligned}
\frac{1-|z|^{2 \Re(\alpha)}}{\Re(\alpha)}\left|\frac{z I_{L_{i}, b, c, \gamma_{i}, \delta_{i}}^{\prime \prime}(z)}{I_{L_{i}, b, c, \gamma_{i}, \delta_{i}}(z)}\right| \leq & \left\{\frac{1-|z|^{2 \Re(\alpha)}}{\Re(\alpha)} \frac{|c|(6 q-|c|)}{3(4 q-|c|)(3 q-|c|)} \sum_{i=1}^{n}\left|\gamma_{i}\right|\right. \\
& +\frac{2 K}{\left.(2 \Re(\alpha)+1)^{\frac{(2 \Re(\alpha)+1)}{2 \Re(\alpha)}} \sum_{i=1}^{n}\left|\delta_{i}\right|\right\} .}
\end{aligned}
$$

Using the inequalities (36) and (39), we get:

$$
\frac{1-|z|^{2 \Re(\alpha)}}{\Re(\alpha)}\left|\frac{z I_{L_{i}, b, c, \gamma_{i}, \delta_{i}}^{\prime \prime}(z)}{I_{L_{i}, b, c, \gamma_{i}, \delta_{i}}^{\prime}(z)}\right|<1 .
$$

Therefore, by using Lemma 5, we get the required result.

Corollary 1. Consider the function $\mathcal{X}_{L_{i}}(z): \mathcal{D} \longrightarrow \mathbb{C}$ defined in the Equation (9). Let $L_{1}, \ldots, L_{n}>-1.75$ $(n \in \mathbb{N})$ and $L=\min \left\{L_{1}, \ldots, L_{n}\right\}$. Furthermore, let the parameter $\gamma_{i}$ be non-zero complex numbers with $\{i=1,2,3, \ldots, n\}$ and if $g_{i} \in \mathcal{A}$ with:

$$
\left|\frac{g_{i}^{\prime \prime}(z)}{g_{i}^{\prime}(z)}\right| \leq K, \quad z \in \mathcal{D},
$$

where $K \simeq 3.05$, and these numbers satisfy the relations:

$$
\frac{1}{\Re(\alpha)}\left(1+\frac{4(3 L+4)}{3\left(24 L^{2}+58 L+35\right)}\right) \sum_{i=1}^{n}\left|\gamma_{i}\right|+\frac{4}{\Re(\alpha)} \sum_{i=1}^{n}\left|\gamma_{i}\right|<1,
$$

when $0<\Re(\alpha)<1$ and for $\Re(\alpha) \geq 1$ :

$$
\left[\frac{1}{\Re(\alpha)}\left(1+\frac{4(3 L+4)}{3\left(24 L^{2}+58 L+35\right)}\right) \sum_{i=1}^{n}\left|\gamma_{i}\right|+4 \sum_{i=1}^{n}\left|\gamma_{i}\right|<1\right] ;
$$

then for every complex number $\beta, \Re(\beta) \geq \Re(\alpha)>0$, the function $H_{L i, b, c, \gamma_{i}, \beta}$ is univalent.

Corollary 2. Consider the function $\mathcal{X}_{L_{i}}$ defined in the Equation (9). Let $L_{1}, \ldots, L_{n}>-1.75(n \in \mathbb{N})$ and $L=\min \left\{L_{1}, \ldots, L_{n}\right\}$. Furthermore, let the parameter $\gamma_{i}, \delta_{i}$ be non-zero complex numbers and if $g_{i} \in \mathcal{A}$ with:

$$
\left|\frac{g_{i}^{\prime \prime}(z)}{g_{i}^{\prime}(z)}\right| \leq K, \quad z \in \mathcal{D}
$$

where $K \simeq 3.05$, and these numbers satisfy the relation:

$$
\frac{1}{\Re(\alpha)} \frac{4(3 L+4)}{3\left(24 L^{2}+58 L+35\right)} \sum_{i=1}^{n}\left|\gamma_{i}\right|+\frac{2 K}{(2 \Re(\alpha) \Re(\gamma)+1)^{\frac{(2 \Re(\alpha)+1)}{2 \Re(\alpha)}}} \sum_{i=1}^{n}\left|\delta_{i}\right|<1 ;
$$

then for every complex number $\beta, \Re(\beta) \geq \Re(\alpha)>0$, the function $I_{L_{i}, b, c, \gamma_{i}, \delta_{i}, \beta}$ is univalent. 
Corollary 3. Consider the function $\mathcal{Y}_{L_{i}}(z): \mathcal{D} \longrightarrow \mathbb{C}$ defined in the Equation (10). Let $L_{1}, \ldots, L_{n}>-1.75$ $(n \in \mathbb{N})$ and $L=\min \left\{L_{1}, L_{2}, \ldots, L_{n}\right\}$. Furthermore, let the parameters $\gamma_{i}$ be non-zero complex numbers with $\{i=1, \ldots, n\}$ and if $g_{i} \in \mathcal{A}$ with:

$$
\left|\frac{g_{i}^{\prime \prime}(z)}{g_{i}^{\prime}(z)}\right| \leq K, \quad z \in \mathcal{D}
$$

where $K \simeq 3.05$, and these numbers satisfy the relations:

$$
\frac{1}{\Re(\alpha)}\left(1+\frac{4(3 L+4)}{3\left(24 L^{2}+58 L+35\right)}\right) \sum_{i=1}^{n}\left|\gamma_{i}\right|+\frac{4}{\Re(\alpha)} \sum_{i=1}^{n}\left|\gamma_{i}\right|<1,
$$

when $0<\Re(\alpha)<1$ and for $\Re(\alpha) \geq 1$ :

$$
\left[\frac{1}{\Re(\alpha)}\left(1+\frac{4(3 L+4)}{3\left(24 L^{2}+58 L+35\right)}\right) \sum_{i=1}^{n}\left|\gamma_{i}\right|+4 \sum_{i=1}^{n}\left|\gamma_{i}\right|<1\right] ;
$$

then for every complex number $\beta, \Re(\beta) \geq \Re(\alpha)>0$, the function $H_{L_{i}, b, c, \gamma_{i}, \beta}$ is univalent.

Corollary 4. Consider the function $\mathcal{Y}_{L_{i}}(z): \mathcal{D} \longrightarrow \mathbb{C}$ defined in the Equation (10). Let $L_{1}, \ldots, L_{n}>-1.75$ $(n \in \mathbb{N})$ and $L=\min \left\{L_{1}, L_{2}, \ldots, L_{n}\right\}$. Furthermore, let the parameter $\gamma_{i}, \delta_{i}$ be non-zero complex numbers and if $g_{i} \in \mathcal{A}$ with:

$$
\left|\frac{g_{i}^{\prime \prime}(z)}{g_{i}^{\prime}(z)}\right| \leq K, \quad z \in \mathcal{D}
$$

where $K \simeq 3.05$, and these numbers satisfy the relation:

$$
\frac{1}{\Re(\alpha) \Re(\gamma)} \frac{4(3 L+4)}{3\left(24 L^{2}+58 L+35\right)} \sum_{i=1}^{n}\left|\gamma_{i}\right|+\frac{2 K}{(2 \Re(\alpha)+1)^{\frac{(2 \Re(\alpha)+1)}{2 \Re(\alpha)}}} \sum_{i=1}^{n}\left|\delta_{i}\right|<1 ;
$$

then for every complex number $\beta, \Re(\beta) \geq \Re(\alpha)>0$, the function $I_{L_{i}, b, c, \gamma_{i}, \delta_{i}, \beta}$ is univalent.

\section{Starlikeness and Uniform Convexity Criteria for the Integral Operator}

In this section, we find the starlikeness and uniform convexity of these integral operators defined by generalized Struve functions.

Theorem 6. Let $L_{1}, \ldots, L_{n}, b \in \mathbb{R}, c \in \mathbb{C}$ and $q_{i}>\frac{7|c|}{24}$ with $q_{i}=L_{i}+\frac{(b+2)}{2}, i=1, \ldots, n$. Let $v_{L_{i}, b, c}$ : $\mathcal{D} \longrightarrow \mathbb{C}$ be defined in the Equation (8). Let the function $g_{i}$ satisfy the condition $\left|\frac{z g_{i}^{\prime}(z)}{g_{i}(z)}\right| \leq M$, where $M$ is a positive integer. Suppose $q=\min \left(q_{1}, \ldots, q_{n}\right)$ and $\gamma_{i}$ are non-zero complex numbers and these numbers satisfy the relation:

$$
\sum_{i=1}^{n}\left\{\left|\gamma_{i}\right|\left(\frac{|c|(6 q-|c|)}{3(4 q-|c|)(3 q-|c|)}+1\right)+\left|\gamma_{i}\right| M\right\}<1
$$

then the function $H_{L_{i}, b, c, \gamma_{i}, 1}$ defined in the Equation (14) is in class $\mathcal{S}^{*}$.

Proof. Consider the function:

$$
H_{L_{i}, b, c, \gamma_{i}, 1}(z)=\int_{0}^{z} \prod_{i=1}^{n}\left(\frac{v_{L_{i}, b, c}(t)}{g_{i}(t)}\right)^{\gamma_{i}} d t
$$

Hence:

$$
1+\frac{z H_{L_{i}, b, c, \gamma_{i}, 1}^{\prime \prime}(z)}{H_{L_{i}, b, c, \gamma_{i}, 1}^{\prime}(z)}=\sum_{i=1}^{n}\left[\gamma_{i}\left(\frac{z v_{L_{i}, b, c}^{\prime}(z)}{v_{L_{i}, b, c}(z)}\right)-\gamma_{i}\left(\frac{z g_{i}^{\prime}(z)}{g_{i}^{\prime}(z)}\right)\right]+1
$$


This implies that:

$$
\left|1+\frac{z H_{L_{i}, b, c, \gamma_{i}, 1}^{\prime \prime}(z)}{H_{L_{i}, b, c, \gamma_{i}, 1}^{\prime}(z)}\right| \leq \sum_{i=1}^{n}\left|\gamma_{i}\right|\left|\frac{z v_{L_{i}, b, c}^{\prime}(z)}{v_{L_{i}, b, c}(z)}\right|+\sum_{i=1}^{n}\left|\gamma_{i}\right|\left|\frac{z g_{i}^{\prime}(z)}{g_{i}^{\prime}(z)}\right|+1
$$

Using Lemma 4(i),

$$
\left|\frac{z v_{L, b, c}^{\prime}(z)}{v_{L, b, c}(z)}-1\right| \leq \frac{|c|(6 q-|c|)}{3(4 q-|c|)(3 q-|c|)}
$$

and:

$$
\left|\frac{z g_{i}^{\prime}(z)}{g_{i}(z)}\right| \leq M
$$

we have:

$$
\left|1+\frac{z H_{L_{i}, b, c, \gamma_{i}, 1}^{\prime \prime}(z)}{H_{L_{i}, b, c, \gamma_{i}, 1}^{\prime}(z)}\right| \leq \sum_{i=1}^{n}\left\{\gamma_{i}\left(\frac{|c|\left(6 q_{i}-|c|\right)}{3\left(4 q_{i}-|c|\right)\left(3 q_{i}-|c|\right)}+1\right)+\gamma_{i} M\right\}+1 .
$$

Since:

$$
\frac{|c|\left(6 q_{i}-|c|\right)}{3\left(4 q_{i}-|c|\right)\left(3 q_{i}-|c|\right)} \leq \frac{|c|(6 q-|c|)}{3(4 q-|c|)(3 q-|c|)}
$$

therefore:

$$
\left|1+\frac{z H_{L_{i}, b, c, \gamma_{i}, 1}^{\prime \prime}(z)}{H_{L_{i}, b, c, \gamma_{i}, 1}^{\prime}(z)}\right| \leq \sum_{i=1}^{n}\left\{\left|\gamma_{i}\right|\left(\frac{|c|(6 q-|c|)}{3(4 q-|c|)(3 q-|c|)}+1\right)+\left|\gamma_{i}\right| M\right\}+1 .
$$

Furthermore,

$$
\sum_{i=1}^{n}\left\{\left|\gamma_{i}\right|\left(\frac{|c|(6 q-|c|)}{3(4 q-|c|)(3 q-|c|)}+1\right)+\left|\gamma_{i}\right| M\right\}<1
$$

implies that:

$$
\left|1+\frac{z H_{L_{i}, b, c, \gamma_{i}, 1}^{\prime \prime}(z)}{H_{L_{i}, b, c, \gamma_{i}, 1}^{\prime}(z)}\right|<2
$$

By using Lemma 2 , the function $H_{L_{i}, b, c, \gamma_{i}, 1} \in \mathcal{S}^{*}$.

Theorem 7. Let $L_{1}, \ldots, L_{n}, b \in \mathbb{R}, c \in \mathbb{C}$ and $q_{i}>\frac{7|c|}{24}$ with $q_{i}=L_{i}+\frac{b+2}{2}, i=1, \ldots, n$. Let $v_{L_{i}, b, c}: \mathcal{D} \longrightarrow$ $\mathbb{C}$ be defined in the Equation (8). Let the function $g_{i}$ satisfy the condition $\left|\frac{z g_{i}^{\prime}(z)}{g_{i}(z)}\right| \leq M$, where $M$ is a positive integer. Suppose $q=\min \left(q_{1}, q_{2}, \ldots, q_{n}\right)$ and $\gamma_{i}$ are non-zero complex numbers and these numbers satisfy the relation:

$$
\sum_{i=1}^{n}\left\{\left|\gamma_{i}\right|\left(\frac{|c|(6 q-|c|)}{3(4 q-|c|)(3 q-|c|)}+1\right)+\left|\gamma_{i}\right| M\right\}<\frac{1}{2}
$$

then the function $H_{L_{i}, b, c, \gamma_{i}, 1} \in \mathcal{U C V}$.

Proof. Consider the function:

$$
H_{L_{i}, b, c, \gamma_{i}, 1}(z)=\int_{0}^{z} \prod_{i=1}^{n}\left(\frac{v_{L_{i}, b, c}(t)}{g_{i}(t)}\right)^{\gamma_{i}} d t
$$

This implies that:

$$
\frac{z H_{L_{i}, b, c, \gamma_{i}, 1}^{\prime \prime}(z)}{H_{L_{i}, b, c, \gamma_{i}, 1}^{\prime}(z)}=\sum_{i=1}^{n}\left[\gamma_{i}\left(\frac{z v_{L_{i}, b, c}^{\prime}(z)}{v_{L_{i}, b, c}(z)}\right)-\gamma_{i}\left(\frac{z g_{i}^{\prime}(z)}{g_{i}^{\prime}(z)}\right)\right] .
$$


Therefore:

$$
\left|\frac{z H_{L_{i}, b, c, \gamma_{i}, 1}^{\prime \prime}(z)}{H_{L_{i}, b, c, \gamma_{i}, 1}^{\prime}(z)}\right| \leq \sum_{i=1}^{n}\left|\gamma_{i}\right|\left|\frac{z v_{L_{i}, b, c}^{\prime}(z)}{v_{L_{i}, b, c}(z)}\right|+\sum_{i=1}^{n}\left|\gamma_{i}\right|\left|\frac{z g_{i}^{\prime}(z)}{g_{i}^{\prime}(z)}\right|,
$$

Using Lemma 4(i):

$$
\left|\frac{z v_{L, b, c}^{\prime}(z)}{v_{L, b, c}(z)}-1\right| \leq \frac{|c|(6 q-|c|)}{3(4 q-|c|)(3 q-|c|)}
$$

and:

$$
\left|\frac{z g_{i}^{\prime}(z)}{g_{i}(z)}\right| \leq M
$$

we have:

$$
\left|\frac{z H_{L_{i}, b, c, \gamma_{i}, 1}^{\prime \prime}(z)}{H_{L_{i}, b, c, \gamma_{i}, 1}^{\prime}(z)}\right| \leq \sum_{i=1}^{n}\left\{\left|\gamma_{i}\right|\left(\frac{|c|\left(6 q_{i}-|c|\right)}{3\left(4 q_{i}-|c|\right)\left(3 q_{i}-|c|\right)}+1\right)+\left|\gamma_{i}\right| M\right\}
$$

Since:

$$
\frac{|c|\left(6 q_{i}-|c|\right)}{3\left(4 q_{i}-|c|\right)\left(3 q_{i}-|c|\right)} \leq \frac{|c|(6 q-|c|)}{3(4 q-|c|)(3 q-|c|)}
$$

therefore:

$$
\left|\frac{z H_{L_{i}, b, c, \gamma_{i}, 1}^{\prime \prime}(z)}{H_{L_{i}, b, c, \gamma_{i}, 1}^{\prime}(z)}\right| \leq \sum_{i=1}^{n}\left\{\left|\gamma_{i}\right|\left(\frac{|c|(6 q-|c|)}{3(4 q-|c|)(3 q-|c|)}+1\right)+\left|\gamma_{i}\right| M\right\} .
$$

Using:

$$
\sum_{i=1}^{n}\left\{\left|\gamma_{i}\right|\left(\frac{|c|(6 q-|c|)}{3(4 q-|c|)(3 q-|c|)}+1\right)+\left|\gamma_{i}\right| M\right\}<\frac{1}{2}
$$

then:

$$
\left|\frac{z H_{L_{i}, b, c, \gamma_{i}, 1}^{\prime \prime}(z)}{H_{L_{i}, b, c, \gamma_{i}, 1}^{\prime}(z)}\right|<\frac{1}{2}
$$

Hence, by using Lemma $3, H_{L_{i}, b, c, \gamma_{i}, 1} \in \mathcal{U C V}$.

Corollary 5. (1) Consider the function $\mathcal{X}_{L_{i}}$ defined in the Equation (9). Let $L_{1}, \ldots, L_{n}>-1.75(n \in \mathbb{N})$, $L=\min \left\{L_{1}, L_{2}, \ldots, L_{n}\right\}$ and the function $g_{i}$ satisfy the condition $\left|\frac{z g_{i}^{\prime}(z)}{g_{i}(z)}\right| \leq M$, where $M$ is a positive integer. Suppose $q=\min \left(q_{1}, q_{2}, \ldots . . q_{n}\right)$ and $\gamma_{i}$ are non-zero complex numbers and these numbers satisfy the inequality:

$$
\sum_{i=1}^{n}\left\{\left|\gamma_{i}\right|\left(\frac{4(3 L+4)}{3\left(24 L^{2}+58 L+35\right)}+1\right)+\left|\gamma_{i}\right| M\right\}<1
$$

then the function $H_{L_{i}, b, c, \gamma_{i}, 1} \in \mathcal{S}^{*}$.

(2) Consider the function $\mathcal{X}_{L_{i}}$ defined as the Equation (9). Let $L_{1}, \ldots, L_{n}>-1.75(n \in \mathbb{N})$, $L=\min \left\{L_{1}, L_{2}, \ldots, L_{n}\right\}$ and the function $g_{i}$ satisfy the condition $\left|\frac{z g_{i}^{\prime}(z)}{g_{i}(z)}\right| \leq M$, where $M$ is a positive integer. Suppose $q=\min \left(q_{1}, q_{2}, \ldots, q_{n}\right)$ and $\gamma_{i}$ are non-zero complex numbers and these numbers satisfy the inequality:

$$
\sum_{i=1}^{n}\left\{\left|\gamma_{i}\right|\left(\frac{8(3 L+4)}{3\left(24 L^{2}+58 L+35\right)}+1\right)+\left|\gamma_{i}\right| M\right\}<\frac{1}{2}
$$

then the function $H_{L_{i}, b, c, \gamma_{i}, 1} \in \mathcal{U C V}$. 
Corollary 6. (1) Consider the function $\mathcal{Y}_{L_{i}}$ defined as the Equation (10). Let $L_{1}, \ldots, L_{n}>-1.75(n \in \mathbb{N})$, $L=\min \left\{L_{1}, L_{2}, \ldots, L_{n}\right\}$ and the function $g_{i}$ satisfy the condition $\left|\frac{z g_{i}^{\prime}(z)}{g_{i}(z)}\right| \leq M$, where $M$ is a positive integer. Suppose $q=\min \left(q_{1}, q_{2}, \ldots . . . q_{n}\right)$ and $\gamma_{i}$ are non-zero complex numbers and these numbers satisfy the inequality:

$$
\sum_{i=1}^{n}\left\{\left|\gamma_{i}\right|\left(\frac{4(3 L+4)}{3\left(24 L^{2}+58 L+35\right)}+1\right)+\left|\gamma_{i}\right| M\right\}<1
$$

then the function $H_{L_{i}, b, c, \gamma_{i}, 1} \in \mathcal{S}^{*}$.

(2) Consider the function $\mathcal{Y}_{L_{i}}$ defined as the Equation (10). Let $L_{1}, \ldots, L_{n}>-1.75(n \in \mathbb{N}), L=$ $\min \left\{L_{1}, L_{2}, \ldots, L_{n}\right\}$ and the function $g_{i}$ satisfy the condition $\left|\frac{z g_{i}^{\prime}(z)}{g_{i}(z)}\right| \leq M$, where $M$ is a positive integer. Suppose $q=\min \left(q_{1}, q_{2}, \ldots, q_{n}\right)$ and $\gamma_{i}$ are non-zero complex numbers and these numbers satisfy the inequality:

$$
\sum_{i=1}^{n}\left\{\left|\gamma_{i}\right|\left(\frac{8(3 L+4)}{3\left(24 L^{2}+58 L+35\right)}+1\right)+\left|\gamma_{i}\right| M\right\}<\frac{1}{2}
$$

then the function $H_{L_{i}, b, c, \gamma_{i}, 1} \in \mathcal{U C V}$.

Author Contributions: Conceptualization, H.M.S. and M.R.; Formal analysis, H.M.S. and S.N.M.; Funding acquisition, S.M.; Investigation, M.R. and N.S.; Methodology, M.R. and N.S.; Supervision, S.N.M.; Validation, S.N.M.; Visualization, S.Z.; Writing—original draft, S.Z.; Writing_-review \& editing, S.Z.

Funding: This work is partially supported by Sarhad University of Science and I.T., Peshawar, Pakistan.

Acknowledgments: The authors are grateful to the referees for their valuable comments, which improved the quality of the work and the presentation of the paper.

Conflicts of Interest: The authors declare no conflict of interest.

\section{References}

1. Goodman, A.W. On uniformly convex functions. Ann. Pol. Math. 1991, 56, 87-92. [CrossRef]

2. Arif, M.; Raza, M. Some properties of an integral operator defined by Bessel function. Acta Univ. Apul. 2011, 26, 69-74.

3. Baricz, A.; Ponnusamy, S. Starlikeness and convexity of generalized Bessel functions. Integral Transf. Spec. Funct. 2010, 21, 641-653. [CrossRef]

4. Baricz, A. Some inequalities involving generalized Bessel functions. Math. Inequal. Appl. 2007, 10, 827-842. [CrossRef]

5. Baricz, A.; Frasin, B.A. Univalence of integral operators involving Bessel functions. Appl. Math. Lett. 2010, 23, 371-376. [CrossRef]

6. Deniz, E.; Orhan, H.; Srivastava, H.M. Some sufficient conditions for univalence of certain families of integral operators involving generalized Bessel functions. Taiwan J. Math. 2011, 15, 883-917.

7. Frasin, B.A. Sufficient conditions for integral operators defined by Bessel functions. J. Math. Inequal. 2010, 4, 301-306. [CrossRef]

8. Zhang, S.; Jin, J.-M. Computation of Special Functions; Wiley Interscience Publication: New York, NY, USA, 1996.

9. Struve, H. Beitrag zur Theorie der Diffraction an Fernrohren. Ann. Phys. Chem. 1882, 17, 1008-1016. [CrossRef]

10. Orhan, H.; Yagmur, N. Geometric properties of generalized Struve function. Analele ştiințtifice ale Universităţıi "Al. I. Cuza" din Iaşi. Matematică 2017, 63, 229-244. [CrossRef]

11. Baricz, A.; Dimitrov, D.K.; Orhan, H.; Yagmur, N. Radii of starlikeness of some special functions. Proc. Am. Math. Soc. 2016, 144, 3355-3367. [CrossRef]

12. Orhan, H.; Yagmur, N. Starlikeness and convexity of generalized Struve functions. Abstr. Appl. Anal. 2013, 2013, 954513.

13. Raza, M.; Yagmur, N. Some properties of a class of analytic functions defined by generalized Struve functions. Turk. J. Math. 2015, 39, 931-944. [CrossRef] 
14. Din, M.U.; Srivastava, H.M.; Raza, M. Univalence of certain integral operators involving generalized Struve functions. Hacet. J. Math. Stat. 2018, 47, 821-833.

15. Hallenbeck, D.J.; Ruscheweyh, S. Subordination by convex functions. Proc. Am. Math. Soc. 1975, 52, $191-195$. [CrossRef]

16. Miller, S.S.; Mocanu, P.T. Univalence of Gaussian and confluent hypergeometric functions. Proc. Am. Math. Soc. 1990, 110, 333-342 [CrossRef]

17. Ravichandran, V. On uniformly convex functions. Ganita 2002, 53, 117-124.

18. Pescar, V. A new generalization of Ahfors and Beckers criterion of univalence. Bull. Malays. Math. Soc. 1996, 19, 53-54.

19. Kudriasov, N.S. Onekotorih priznakah odnolistnosti analiticesschihfunktii. Mathmaticskie Zametki 1973, 13, 359-366.

(C) 2019 by the authors. Licensee MDPI, Basel, Switzerland. This article is an open access article distributed under the terms and conditions of the Creative Commons Attribution (CC BY) license (http://creativecommons.org/licenses/by/4.0/). 